\title{
Unusual development of light-reflecting pigment cells in intact and regenerating tail in the periodic albino mutant of Xenopus laevis
}

\author{
Toshihiko Fukuzawa
}

Received: 7 June 2010 / Accepted: 9 August 2010 /Published online: 22 September 2010

(C) The Author(s) 2010. This article is published with open access at Springerlink.com

\begin{abstract}
Unusual light-reflecting pigment cells, "white pigment cells", specifically appear in the periodic albino mutant $\left(a^{p} / a^{p}\right)$ of Xenopus laevis and localize in the same place where melanophores normally differentiate in the wild-type. The mechanism responsible for the development of unusual pigment cells is unclear. In this study, white pigment cells in the periodic albino were compared with melanophores in the wild-type, using a cell culture system and a tail-regenerating system. Observations of both intact and cultured cells demonstrate that white pigment cells are unique in (1) showing characteristics of melanophore precursors at various stages of development, (2) accumulating reflecting platelets characteristic of iridophores, and (3) exhibiting pigment dispersion in response to $\alpha$-melanocyte stimulating hormone $(\alpha-\mathrm{MSH})$ in the same way that melanophores do. When a tadpole tail is amputated, a functionally competent new tail is regenerated. White pigment cells appear in the mutant regenerating tail, whereas melanophores differentiate in the wild-type regenerating tail. White pigment cells in the mutant regenerating tail are essentially similar to melanophores in the wild-type regenerating tail with respect to their localization, number, and response to $\alpha$-MSH. In addition to white pigment cells, iridophores which are never present in the intact tadpole tail appear specifically in the somites near the amputation level in the mutant regenerating tail. Iridophores are distinct from white pigment cells in size, shape, blue light-induced fluorescence, and response to $\alpha$ MSH. These findings strongly suggest that white pigment cells in the mutant arise from melanophore precursors and accumulate reflecting platelets characteristic of iridophores.
\end{abstract}

\section{T. Fukuzawa $(\bowtie)$}

Department of Biology, Keio University,

Hiyoshi 4-1-1, Kohoku-ku,

Yokohama 223-8521, Japan

e-mail: fukuzawa@fbc.keio.ac.jp
Keywords Periodic albino - Melanophore - Iridophore Regeneration $\cdot$ Xenopus laevis (Anura)

\section{Introduction}

Pigment cells derive from neural crest cells in vertebrates (Bagnara and Hadley 1973; Hall and Hörstadius 1988; Le Douarin and Kalcheim 1999). In poikilotherms, a wide variety of pigment cells are known. They are melanophores (brown or black due to melanin in melanosomes), iridophores (silver or gold due to reflecting platelets), leucophores (white due to leucosomes), xanthophores (yellow to orange due to pterinosomes and/or carotenoid vesicles), erythrophores (orange to red due to pterinosomes and/or carotenoid vesicles), and cyanophores (blue due to cyanosomes) (Bagnara 1998). Recently, studies have been made on genetic regulation of melanophore development using zebrafish (Elworthy et al. 2003; Kelsh et al. 2000, 2009; Lister et al. 1999; Parichy et al. 1999, 2000a; Rawls and Johnson 2003). Genetic regulation of xanthophore development has been also analyzed in zebrafish and medaka (Fukamachi et al. 2006; Minchin and Hughes 2008; Parichy et al. 2000b). On the other hand, the mechanism is not clear as to how differentiation and pigment organellogenesis are controlled in light-reflecting pigment cells, although several genes have been suggested to be required for iridophore development (Lister et al. 2006; Lopes et al. 2008).

The periodic albino mutant $\left(a^{p} / a^{p}\right)$ of Xenopus laevis shows interesting characteristics of pigmentation and pigment cell differentiation (Fukuzawa 2004; Fukuzawa and Ide 1986, 1987; Hoperskaya 1975, 1981; MacMillan 1979, 1981). This mutant is characterized by the absence of melanin in oocytes, the appearance of melanin in the pigment epithelium of the eye and in skin melanophores at 
larval stages, and the almost complete disappearance of melanin in metamorphosed animals (Hoperskaya 1975). Ultrastructural observation has revealed that melanophores in this mutant contain many abnormal melanosomes with granular internal structures (Fukuzawa and Ide 1986; Hoperskaya 1981; Seldenrijk et al. 1982). It has been shown that iridophores (Fukuzawa 2006; MacMillan 1979; MacMillan and Gordon 1981) and xanthophores (Fukuzawa 2006) are also affected in the periodic albino. Recently, the author has reported that unusual light-reflecting pigment cells, which show the characteristic features of both melanophores and iridophores, specifically appear in the periodic albino mutant (Fukuzawa 2004). These cells were previously called "leucophore-like cells", because they resemble leucophores in the fish, Oryzias latipes, with respect to color, blue lightinduced fluorescence, and response to $\alpha$-MSH (Fukuzawa 2004). Indeed, "leucophore-like cells" are unusual in (1) looking white under incident light, (2) emitting green fluorescence under blue light, (3) exhibiting pigment dispersion in the presence of $\alpha$-melanocyte stimulating hormone ( $\alpha$-MSH), and (4) containing many premelanosomes and reflecting platelet-like organelles (Fukuzawa 2004). So far, the mechanism responsible for the development of unusual light-reflecting pigment cells is not known.

In this paper, "leucophore-like cells" in the periodic albino mutant are called "white pigment cells", because the term "leucophore" may be confusing or misleading in discussing the origin of these cells. The present study was designed to elucidate how light-reflecting pigment cells, especially white pigment cells, originate in the periodic albino. To examine the origin of white pigment cells in the mutant, these cells were compared with melanophores in the wild-type, using a cell culture system and a tail-regenerating system.

The Xenopus tadpole tail is best suited for the purpose of this study, because only white pigment cells are present in the posterior region of the mutant tadpole tail, whereas only melanophores localize in the same region of the wild-type tadpole. Therefore, white pigment cells of the mutant tadpole tail were compared with melanophores of the wild-type tadpole tail. In some experiments, differentiation from neural crest cells to pigment cells was compared between the wildtype and the mutant in the neural crest cell culture system utilizing serum-free medium. Melanophore precursors were confirmed by dopa reaction, combined dopa-premelanin reaction, and electron microscopy. Observations of both intact and cultured cells indicate that (1) white pigment cells show characteristics of melanophore precursors at various stages of development, (2) white pigment cells accumulate reflecting platelets characteristic of iridophores, and (3) white pigment cells exhibit pigment dispersion in response to $\alpha$-MSH in the same way that melanophores do.

Another advantage of utilizing the Xenopus tadpole tail to investigate pigment cell development lies in the fact that the
Xenopus tadpole tail is an ideal model for studying regeneration of a variety of cells (Beck et al. 2009; Slack et al. 2008; Tseng and Levin 2008). Indeed, the study of regeneration of neural crest derivatives has shown that melanophores arise from pre-existing melanophore precursors in the wild-type regenerating tail (Lin et al. 2007). Accordingly, pigment cells which appeared in the mutant regenerating tail were compared with melanophores in the wild-type regenerating tail. When the tadpole tail is amputated, a functionally competent new tail is regenerated in both the wild-type and the mutant $X$. laevis. White pigment cells appear in the mutant regenerating tail, while melanophores differentiate in the wild-type regenerating tail. White pigment cells in the mutant regenerating tail are essentially similar to melanophores in the wild-type regenerating tail with respect to their localization, number, and response to $\alpha-\mathrm{MSH}$. Interestingly, dopa staining was observed in both white pigment cells and melanophore precursors in the mutant regenerating tail. In addition to white pigment cells, a small number of iridophores which are never present in the intact tadpole tail appear specifically in the somites near the amputation level in the mutant regenerating tail. Iridophores are distinct from white pigment cells in size, shape, blue light-induced fluorescence, and response to $\alpha-\mathrm{MSH}$.

The present findings, together with previous reports (Fukuzawa 2004, 2006), strongly suggest that white pigment cells arise from melanophore precursors and accumulate reflecting platelets in the periodic albino mutant. Development of light-reflecting pigment cells is discussed.

\section{Materials and methods}

Wild-type (+/+) and periodic albino mutant $\left(a^{p} / a^{p}\right)$ of Xenopus laevis were used in the present experiments. Xenopus eggs were obtained by gonadotropin stimulation, and developmental stages were determined according to Nieuwkoop and Faber (1967).

Culture of neural crest cells using serum free medium

Neural tubes of wild-type and mutant embryos (stage 22) were used as the source of neural crest cells as described previously (Fukuzawa and Ide 1988; Fukuzawa and Bagnara 1989). The epidermis, somites, and notochord were removed from Xenopus embryos after $0.1 \%$ collagenase treatment for $30 \mathrm{~min}$. The neural tube was then cut with a tungsten needle at the level of the seventh somite, and the caudal half of the neural tube was cultured in a sitting drop of $70 \mu \mathrm{l}$ of culture medium on a tissue culture dish (Falcon 3001; Becton Dickinson, Franklin Lakes, NJ, 
USA) at $25^{\circ} \mathrm{C}$. In this experiment, a serum-free medium was used, which was a mixture of 7 parts of Leibovitz's L-15 (Gibco, Grand Island, NY, USA), 3 parts of Milli-Q ultrapure water (Millipore, Tokyo, Japan), $20 \mu \mathrm{g} / \mathrm{ml}$ transferrin (Biomedical Technologies, Stoughton, MA, USA), and $20 \mu \mathrm{g} / \mathrm{ml}$ insulin (Cell Science \& Technology Institute, Sendai, Japan). Neural tubes attach to the culture dishes within $10 \mathrm{~h}$, and neural crest cells begin to migrate out from them. After 2 days, $2 \mathrm{ml}$ of the medium was added to the culture. Subsequently, the medium was changed every 5 days. To check the effect of serum on melanophore differentiation, neural crest cells were also cultured in a serum-supplemented medium, which consisted of 5 parts of Leibovitz'sL-15 (Gibco), 3 parts of Milli-Q ultrapure water (Millipore), and 2 parts of fetal calf serum (Gibco) (Fukuzawa 2004).

Culture of white pigment cells in the periodic albino mutant

To isolate white pigment cells, tails of mutant tadpoles (stage 52) were utilized. Tadpole tails were cut and washed with sterile Steinberg's balanced salt solution (BSS; Jones and Elsdale 1963). Then, the tails were chopped with scissors and dissociated with $0.3 \%$ collagenase for $30 \mathrm{~min}$. The resultant cell suspension was collected, washed, and cultured at $25^{\circ} \mathrm{C}$ in a tissue culture dish (Falcon 3001; Becton Dickinson) using the same culture medium that was utilized for the culture of neural crest cells.

\section{$\alpha$-MSH stimulation in pigment cells}

The effect of $\alpha$-MSH was compared between melanophores of the wild-type and white pigment cells of the mutant. Excised tadpole tails (stage 48) or cultured white pigment cells in the mutant were placed in Steinberg's BSS until pigment organelles aggregated within pigment cells. Then, $\alpha$-MSH (Calbiochem, San Diego, CA, USA) $(1 \mu \mathrm{g} / \mathrm{ml})$ was added to them, and the response of the pigment cells to $\alpha$ $\mathrm{MSH}$ was monitored on an inverted microscope (Olympus IX71; Japan). In some experiments, $\alpha$-MSH $(1 \mu \mathrm{g} / \mathrm{ml})$ was added and tested on iridophores which appeared in the regenerating tail of the mutant.

Tail regeneration experiments

Wild-type and mutant tadpoles (stage 48-50) were utilized for tail regeneration experiments. The distal $50 \%$ of the tail was amputated with a sharp razor blade in Steinberg's BSS. The tadpoles were healed and reared in 10\% Steinberg's BSS. After amputation, the regenerating tail was photographed every day. The number of pigment cells which appeared in the regenerating tail was counted and compared between the wild-type and the mutant.
Dopa reaction and dopa-premelanin reaction

Cultured neural crest cells and the regenerating tails were fixed with $15 \%$ formalin in $0.1 \mathrm{M}$ phosphate buffer $(\mathrm{pH} 7.4)$ at $4^{\circ} \mathrm{C}$ for $24 \mathrm{~h}$, washed with the same buffer, and incubated with $0.1 \%$ 3,4-dihydroxyphenylalanin (L-dopa) in $0.1 \mathrm{M}$ phosphate buffer $(\mathrm{pH} 7.4)$ at $37^{\circ} \mathrm{C}$ for $12 \mathrm{~h}$. They were refixed in $10 \%$ formalin and used for observation (Fukuzawa and Ide 1986). To detect melanophore precursor cells with stage I and II premelanosomes, in addition to tyrosinase positive cells, combined dopa-premelanin reaction (combined dopaammoniacal silver nitrate staining) was performed (Mishima 1960; Hirobe and Takeuchi 1977; Hirobe 1984). Cultured neural crest cells after dopa treatment were further incubated with $10 \%$ ammoniacal silver nitrate (Wako Pure Chemical Industries, Osaka, Japan) for $10 \mathrm{~min}$ at $58^{\circ} \mathrm{C}$ (Mishima 1960; Hirobe and Takeuchi 1977; Hirobe 1984) and were used for observation.

\section{Fluorescence microscopy}

Both intact and regenerating tails were observed with a fluorescence stereomicroscope (Leica MZ FL III, Wetzlar, Germany), while an inverted microscope (Olympus IX71) was utilized to observe cultured neural crest cells and pigment cells in vitro. To check pigment cells, observations were made with transmitted light, incident illumination, and blue light (460-500 $\mathrm{nm})$.

\section{Electron microscopy}

Melanoblasts, melanophores, iridophores, and white pigment cells which appeared in the periodic albino mutant were examined electron microscopically to identify pigment organelles. Both intact and cultured cells were fixed in $2.5 \%$ glutaraldehyde in $0.1 \mathrm{M}$ cacodylate buffer $(\mathrm{pH} 7.2)$ for $60 \mathrm{~min}$ at $4^{\circ} \mathrm{C}$, post-fixed in $2 \%$ $\mathrm{O}_{\mathrm{S}} \mathrm{O}_{4}$ in the same buffer for $60 \mathrm{~min}$ at $4^{\circ} \mathrm{C}$, dehydrated through a graded series of ethanol and embedded in epoxy resin. Ultrathin sections were stained with uranyl acetate and lead citrate, and observed on a JEOL JEM-1010 electron microscope.

\section{Results}

White pigment cells appeared specifically in the periodic albino mutant, but not in the wild-type

Among the three types of pigment cells (melanophores, xanthophores, and iridophores), melanophores differentiated first, and xanthophores and iridophores appeared later in the development of Xenopus laevis. Only 
melanophores localized in the posterior part of the wildtype tadpole tail (Fig. 1a, b). Wild-type melanophores containing many mature melanosomes (Fig. 1c) looked black, and dispersed melanosomes in response to $\alpha$-MSH $(1 \mu \mathrm{g} / \mathrm{ml})$ (Fig. 1a, b). In contrast, white pigment cells, instead of melanophores, specifically appeared in the posterior part of the mutant tadpole tail (Fig. 1d, e), and were similar in density to melanophores of the wild-type tadpole tail (Fig. 1a, b). White pigment cells in the mutant reflected light and looked white under incident light, and dispersed pigment organelles in response to $\alpha$-MSH (Fig. 1d, e) in the same manner as wild-type melanophores (Fig. 1a, b). White pigment cells retained characteristics of both melanophores and iridophores, because they contained premelanosomes characteristic of melanophores and reflecting platelets characteristic of iridophores, although reflecting platelets in white pigment cells were irregular in size and shape (Fig. 1f).
Melanophore precursors were maintained without differentiating into melanophores in the mutant neural crest cell culture using serum-free medium

In both the wild-type and the mutant, neural tube explants attached to the culture dishes within $10 \mathrm{~h}$, and neural crest cells migrated out from them (Figs. 2, 3, 4). Melanophores differentiated from wild-type neural crest cells in culture using either serum-free medium (Fig. 2a-d) or serum-supplemented medium (Fukuzawa 2004). Mutant melanophores also differentiated in serum-supplemented medium (Fig. 3a); however, mutant neural crest cells were unmelanized in serum-free medium (Figs. $3 \mathrm{~d}$ and $4 \mathrm{a}, \mathrm{b}$ ). These unmelanized cells in serum-free medium were identified as melanophore precursors at various stages of development by dopa reaction (Fig. 3e) and combined dopa-premelanin reaction (Fig. 3f), and were discriminated from differentiated melanophores with visible melanosomes
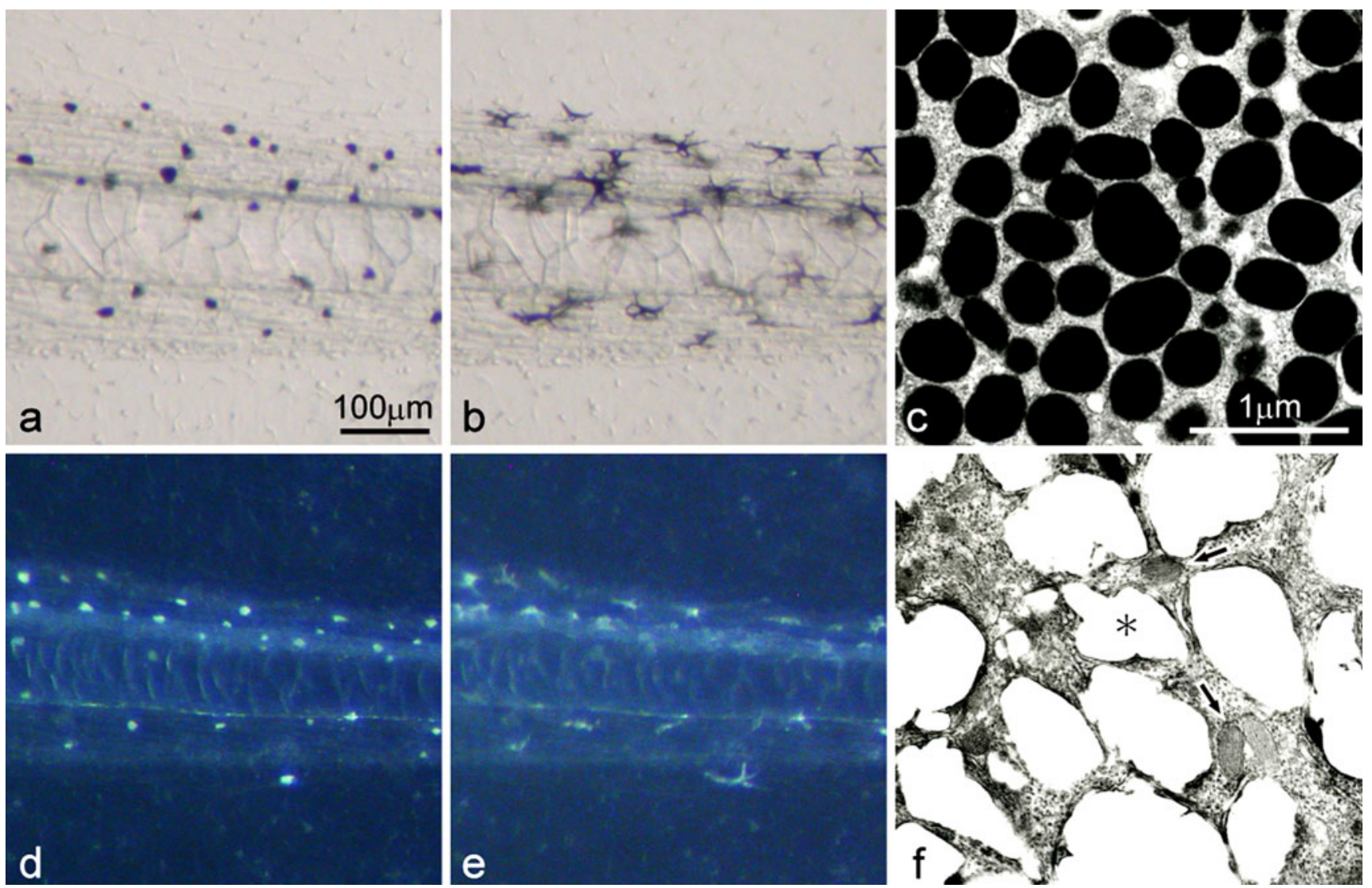

Fig. 1 Pigment cells present in the wild-type tadpole tail $(\mathbf{a}-\mathbf{c})$ and the mutant tadpole tail $(\mathbf{d}-\mathbf{f})$ at stage 48 . a The wild-type tail placed in BSS before $\alpha$-MSH administration (transmitted light). b The wild-type tail after $\alpha$-MSH administration $(1 \mu \mathrm{g} / \mathrm{ml})$ (transmitted light). c Ultrastructure of melanophores of the wild-type. d The mutant tail placed in BSS before $\alpha$-MSH administration (incident light). e The mutant tail after $\alpha$-MSH administration $(1 \mu \mathrm{g} / \mathrm{ml})$ (incident light). f

Ultrastructure of white pigment cells in the mutant. While wild-type melanophores were filled with many mature melanosomes (c), white pigment cells in the mutant contained both irregular reflecting platelets (f, asterisk) and premelanosomes with internal lamellar structures (f, arrows). Note that white pigment cells (d,e) in the mutant responded to $\alpha$-MSH and dispersed pigment organelles in the same manner as wildtype melanophores (a b) 


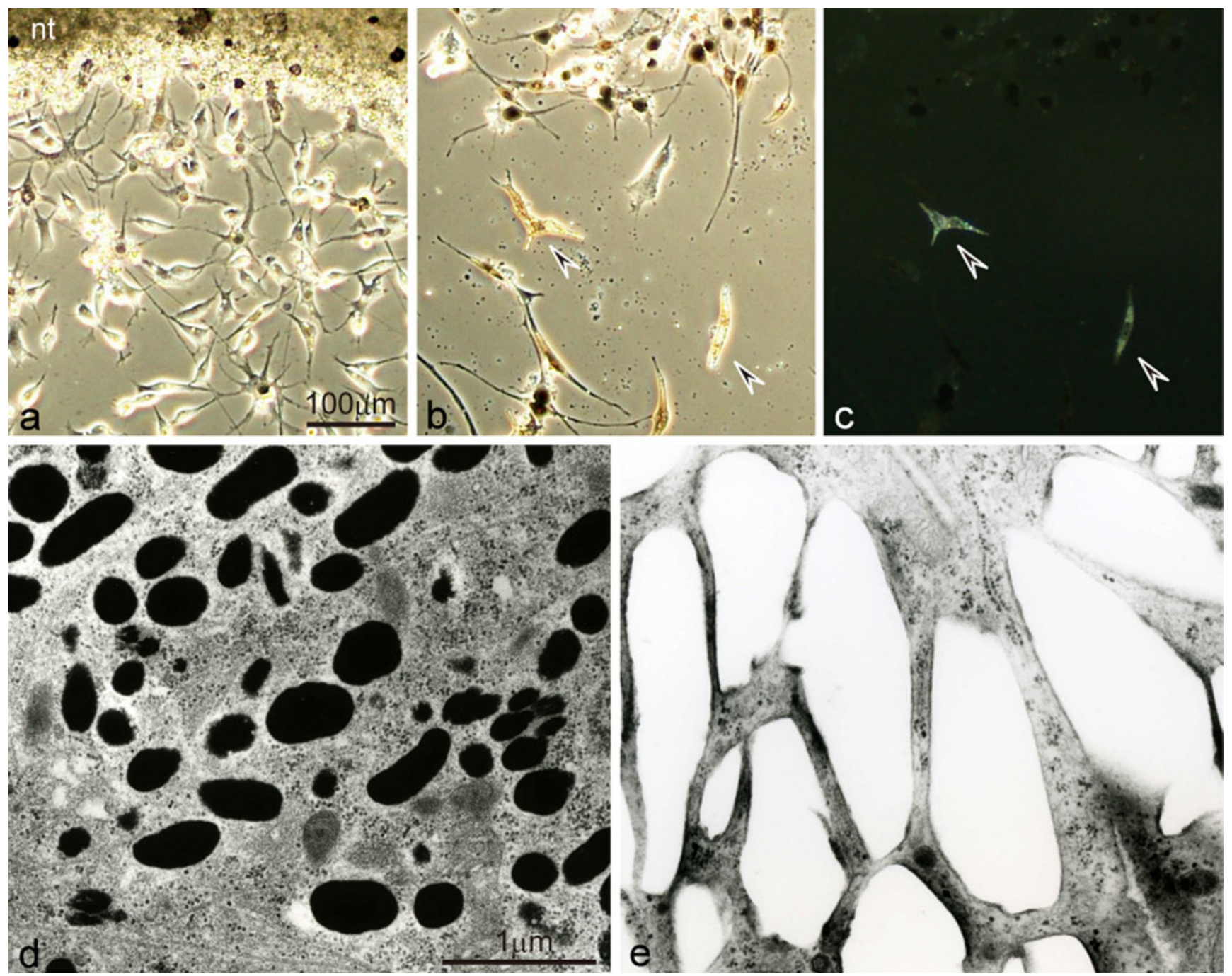

Fig. 2 Differentiation of pigment cells from wild-type neural crest cells in culture using serum-free medium. a Differentiating melanophores and neural crest cells migrating out from the neural tube explant (nt) after 3 days in culture. b,c Differentiated melanophores and iridophores after 20 days in culture observed under transmitted light (b), or incident light (c). d,e Ultrastructure of differentiated melanophores (d) and iridophores (e) in culture. Wild-type melanophores, which differentiated first in culture, were dendritic and

(Fig. 3a) which were further stained with dopa (Fig. 3b) and ammoniacal silver nitrate (Fig. 3c). Unmelanized cells stained with ammoniacal silver nitrate but not with dopa (Fig. $3 \mathrm{~d}-\mathrm{f}$, asterisks) were thought to be melanophore precursors with unmelanized premelanosomes, while those stained with dopa (Fig. 3d-f, arrows) were identified as melanophore precursors at the late stage of development. Ultrastructural observation confirmed that melanophore precursors at different stages of development were maintained without differentiating into melanophores in the periodic albino in serum-free medium (Fig. $4 d-f$ ). Mutant melanophore precursors at early to middle stages of development contained stage I and II premelanosomes aggregated melanosomes (a,b). Note that melanophores also appeared on the neural tube explant (a). Wild-type iridophores, which differentiated later in culture, looked brown under transmitted light (b, arrowheads) and reflected light under incident illumination (c, arrowheads). Wild-type melanophores contained many melanosomes (d), while wild-type iridophores were filled with many rectangular reflecting platelets (e)

(Fig. 4d) according to the mammalian nomenclature of developing melanosomes (reviewed by Raposo and Marks 2007). On the other hand, mutant melanophore precursors at the late stage of development contained stage II premelanosomes and a small number of partially melanized stage III melanosomes (Fig. 4f). The number of premelanosomes varied among melanophore precursors (Fig. $4 \mathrm{~d}-\mathrm{f}$ ).

Iridophores differentiated from both wild-type and mutant neural crest cells in culture using serum-free medium

Iridophores appeared only on the neural tube explants, but not among neural crest cells migrating out from the 


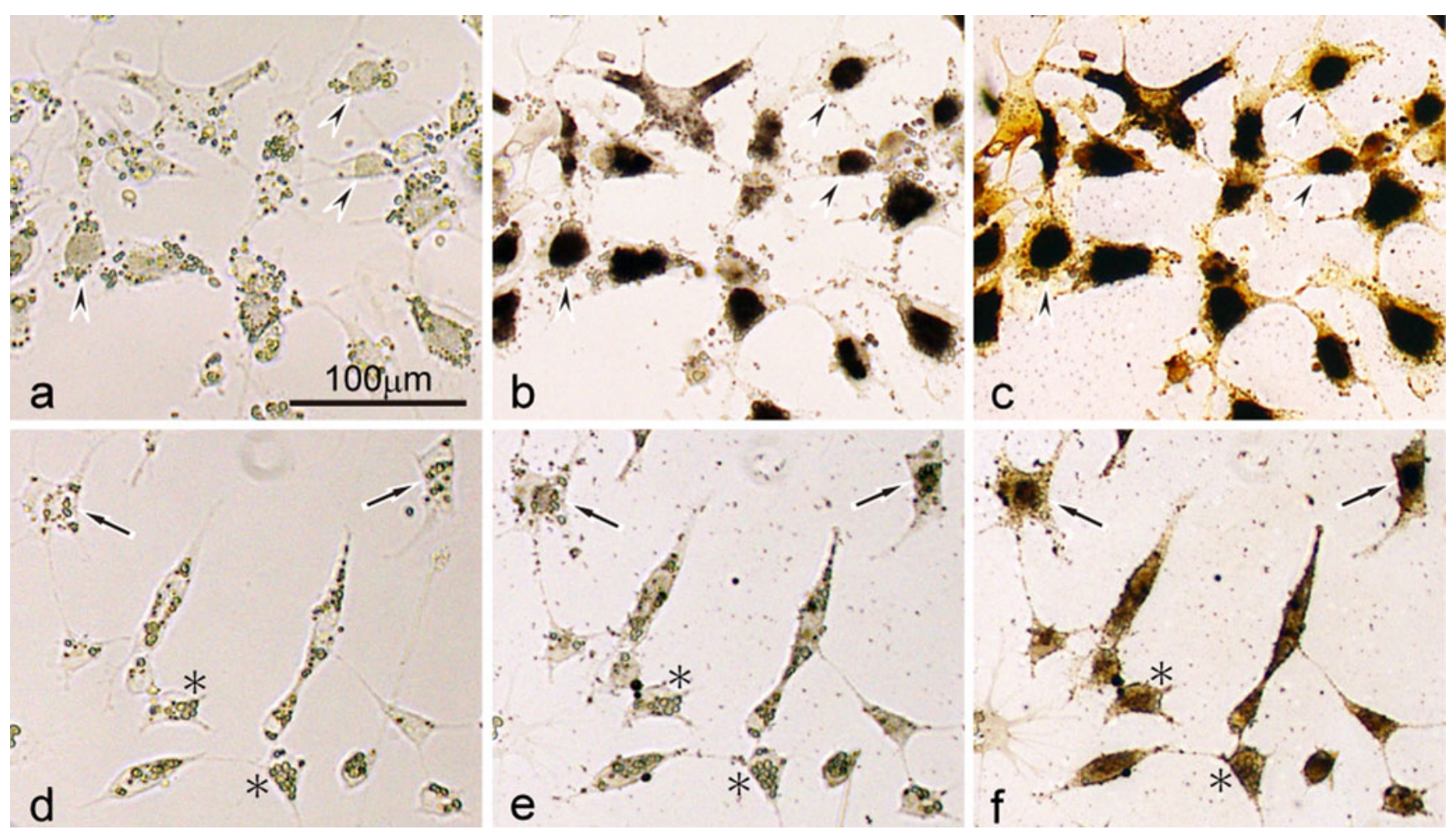

Fig. 3 Dopa and ammoniacal silver nitrate staining in mutant neural crest cell culture. a-c Differentiating melanophores in serumsupplemented medium on day 3 before dopa staining (a), and after dopa (b) and subsequent ammoniacal silver nitrate staining (c). d-f Melanophore precursors in serum-free medium on day 3 before dopa staining (d), and after dopa (e) and subsequent ammoniacal silver nitrate staining (f). Mutant melanophores differentiated in serumsupplemented medium and contained visible melanosomes (a). These melanophores showed strong dopa staining as well as ammoniacal silver nitrate staining (arrowheads). On the other hand, mutant neural crest cells were unmelanized in serum-free medium (d). These unmelanized cells were identified as melanophore precursors at various stages of development, because some cells were stained with both dopa and ammoniacal silver nitrate (arrows), whereas the other cells were stained with ammoniacal silver nitrate, but not with dopa (asterisks). The round bodies within the cells are yolk platelets explants, when medium containing fetal calf serum was used for culture (Fukuzawa 2006). In contrast, iridophores differentiated from migrated neural crest cells in culture using serum-free medium in both the wild-type and the mutant (Figs. 2 and 4). Wild-type iridophores reflected light and looked iridescent gold under incident light (Fig. 2b, c), and contained many rectangular reflecting platelets (Fig. 2e). Mutant iridophores reflected light and looked silver or whitish-gray (Fig. 4b, c) as described previously (Fukuzawa 2006). Reflecting platelets of mutant iridophores (Fig. 4g) were different from those of wildtype iridophores in size and shape. White pigment cells did not appear in wild-type and mutant neural crest cell culture regardless of the presence or absence of fetal calf serum in the medium.

White pigment cells cultured from mutant tadpole tails showed characteristics of melanophore precursors at various stages of development

White pigment cells were isolated and cultured from mutant tadpole tails (stage 52) using serum-free medium, because isolated cells were suited for physiological and ultrastructural analysis. White pigment cells, which were punctuate in culture, looked brown under transmitted light (Fig. 5a), but white under incident illumination (Fig. 5b). Isolated white pigment cells in vitro, which had a few dendrites, responded to $\alpha$-MSH $(1 \mu \mathrm{g} /$

Fig. 4 Differentiation of pigment cells from mutant neural crest cells in culture using serum-free medium. a Neural crest cells migrating out from the neural tube explant $(n t)$ after 3 days in culture. b,c Differentiated iridophores after 20 days in culture observed under transmitted light (b), or incident light (c). d-g Ultrastructure of melanophore precursors at various stages of development $(\mathbf{d}-\mathbf{f})$ and iridophores (g) in culture. In contrast to wild-type neural crest cells, melanophores did not differentiate from mutant neural crest cells in culture using serum-free medium $(\mathbf{a}, \mathbf{b})$. In the present culture condition, melanophore precursors at various stages of development were detected in the mutant $(\mathbf{d}-\mathbf{f})$. Mutant melanophore precursors at early to middle stages of development (d) contained stage I premelanosomes $(\mathrm{ml})$ and stage II premelanosomes $(\mathrm{m} 2)$. In contrast, mutant melanophore precursors at the late stage of development (f) contained stage II premelanosomes and a small number of partially melanized stage III melanosomes $(\mathrm{m} 3)$. The number of premelanosomes varied among melanophore precursors $(\mathbf{d}-\mathbf{f})$. Mutant iridophores differentiated in culture (b,c, arrowheads) in the same manner as wild-type iridophores. However, reflecting platelets of mutant iridophores were irregular in size and shape (g, asterisk) 


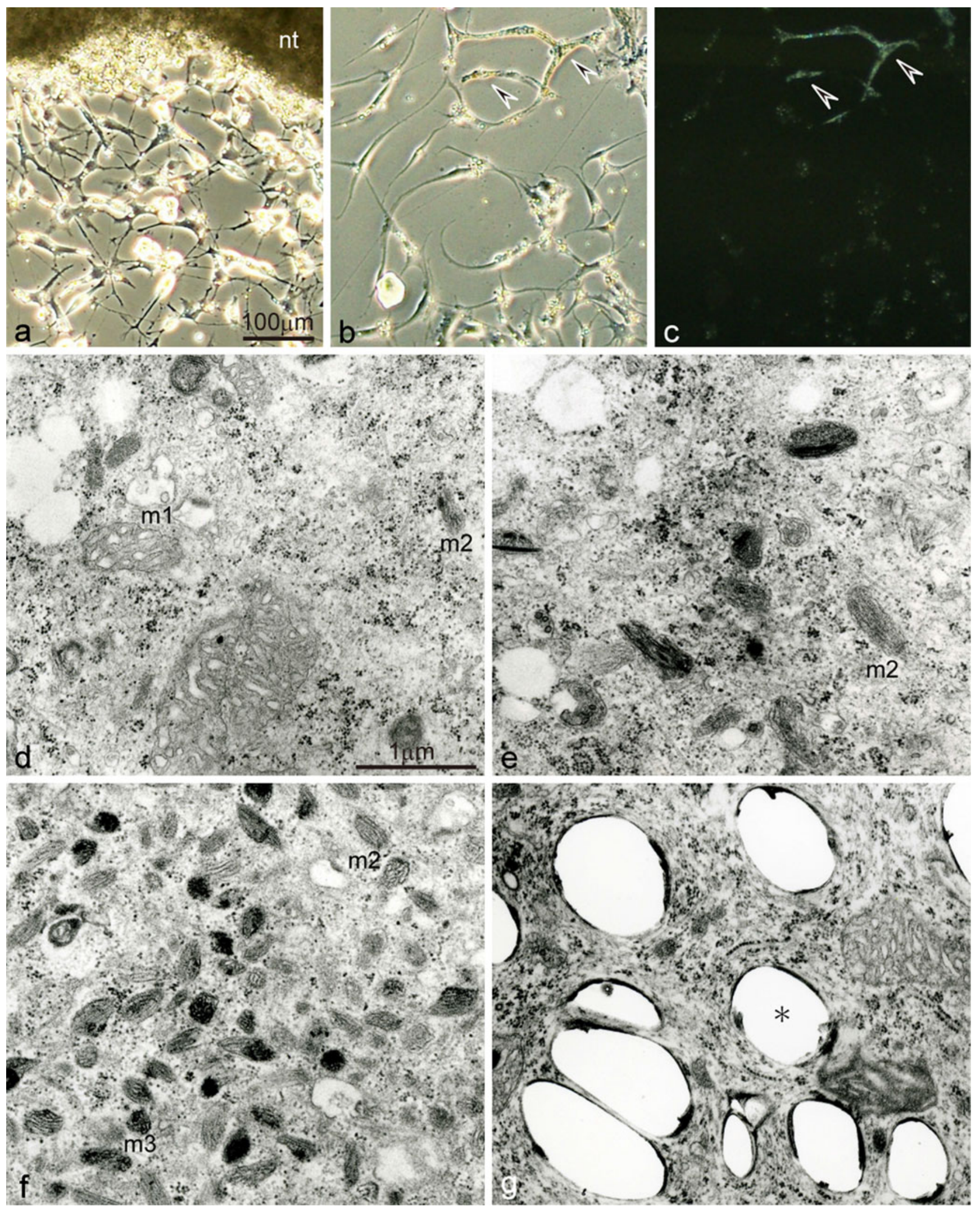




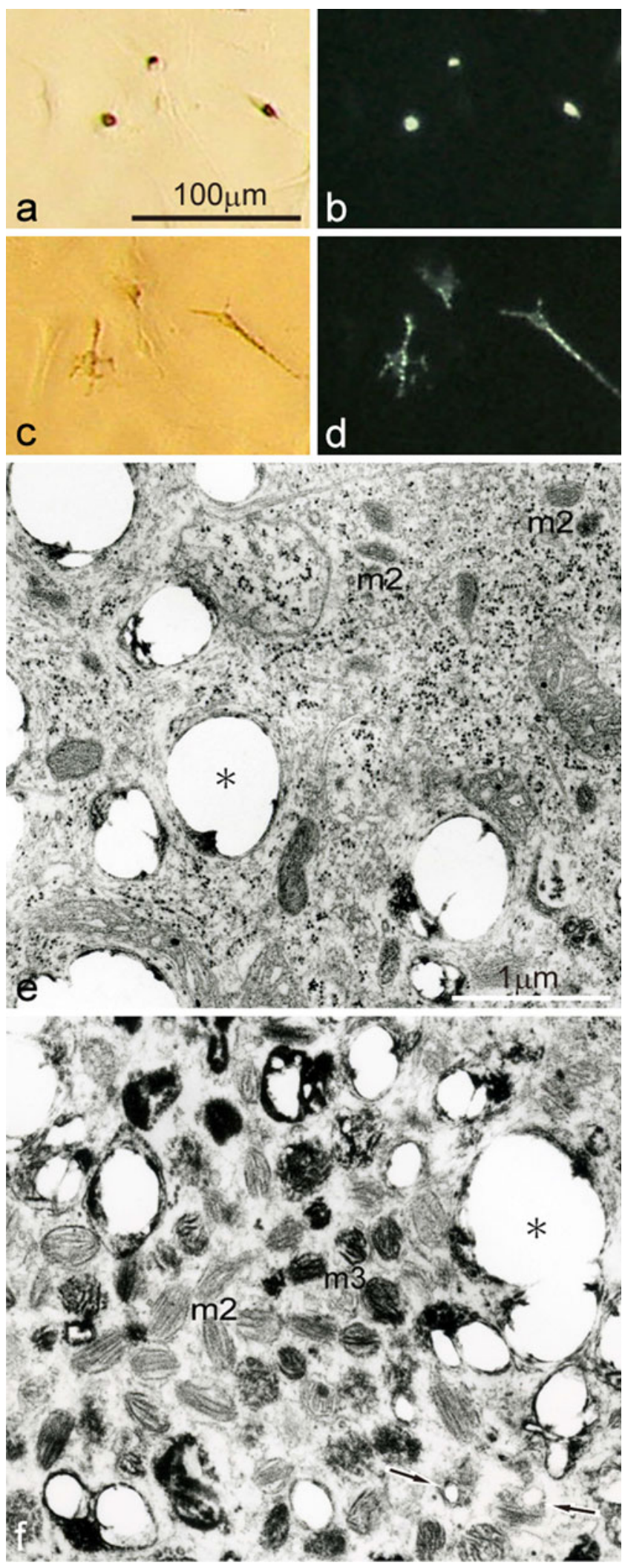

Fig. 5 Physiological and ultrastructural characteristics of white pigment cells which were cultured from mutant tadpole tails at stage 52. a,b Cultured white pigment cells before $\alpha$-MSH administration observed under transmitted light (a), or incident light (b). c,d The same fields as (a) and (b), respectively, after $\alpha$-MSH administration $(1 \mu \mathrm{g} / \mathrm{ml})$ observed under transmitted light (c), or incident light (d). e,f Ultrastructural variation of white pigment cells in culture. White pigment cells which had a few dendrites dispersed pigment organelles in response to $\alpha-\mathrm{MSH}(\mathbf{a}-\mathbf{d})$. Some white pigment cells (e) contained irregular reflecting platelets (asterisk), in addition to stage II premelanosomes $(\mathrm{m} 2)$, whose organelles were characteristic of melanophore precursors at early to middle stages of development. The other white pigment cells (f) contained irregular reflecting platelets (asterisk), in addition to stage II premelanosomes and a small number of partially melanized stage III melanosomes $(\mathrm{m} 3)$, whose organelles were characteristic of melanophore precursors at the late stage of development. Arrows indicate premelanosomes in which reflecting platelet formation seems to be occurring

$\mathrm{ml}$ ) and dispersed pigment organelles (Fig. 5c, d) in the same manner as white pigment cells in the intact tadpole tail of the mutant (Fig. 1d, e). Electron-microscopic observation demonstrated that white pigment cells contained not only irregular reflecting platelets but also organelles showing characteristics of melanophore precursors at various stages of development (Fig. 5e, f). Indeed, some white pigment cells contained stage II premelanosomes which were characteristic of melanophore precursors at early to middle stages of development (Fig. 5e), while the other white pigment cells had stage II premelanosomes and a small number of partially melanized stage III melanosomes, which were characteristic of melanophore precursors at the late stage of development (Fig. 5f). It should be noted that some white pigment cells in the mutant tadpole tail were reported to contain $a^{p}$-type melanosomes, which were characteristic of differentiated mutant melanophores (Fukuzawa 2004). On the other hand, the number of reflecting platelets contained in white pigment cells varied. Some white pigment cells contained many reflecting platelets, whereas the number of reflecting platelets was relatively small in other white pigment cells. In the electron-microscopic image of white pigment cells shown in Fig. 5, reflecting platelet formation seems to be occurring in some premelanosomes (Fig. 5f, arrows). The presence or absence of fetal calf serum in the culture medium did not affect physiological and ultrastructural characteristics of white pigment cells.

Abundant white pigment cells and a small number of iridophores appeared in the mutant regenerating tail, but not in the wild-type regenerating tail

When a tadpole tail (stage 48-50) was amputated, a functionally competent new tail was regenerated in both the wild-type and the mutant (Fig. 6). Melanophores, which were normally present in the posterior part of the wild-type tadpole tail, appeared in the wild-type regenerating tail. Melanophores were detected in the wild-type regenerating 
Fig. 6 Expression of pigment cells in the 6-day regenerating tail in the wild-type and the mutant (amputated at stage 50). a,b The wild-type regenerating tail observed under transmitted light (a) or incident light (b). c,d The mutant regenerating tail observed under transmitted light (c) or incident light (d). e Ultrastructure of differentiated iridophores in the mutant regenerating tail. Dashed lines indicate the amputation level. Melanophores appeared in the wild-type regenerating tail $(\mathbf{a}, \mathbf{b})$, and their distribution was similar to that in the intact tadpole tail. In contrast, white pigment cells (arrows) appeared in the mutant regenerating tail (c,d), and their distribution was similar to that in the intact tadpole tail. A small number of iridophores (arrowheads), which were never present in the intact tadpole tail, appeared in the somites of the mutant regenerating tail in addition to white pigment cells. Differentiated iridophores in the mutant regenerating tail were filled with reflecting platelets, which were irregular in size and shape (asterisk)
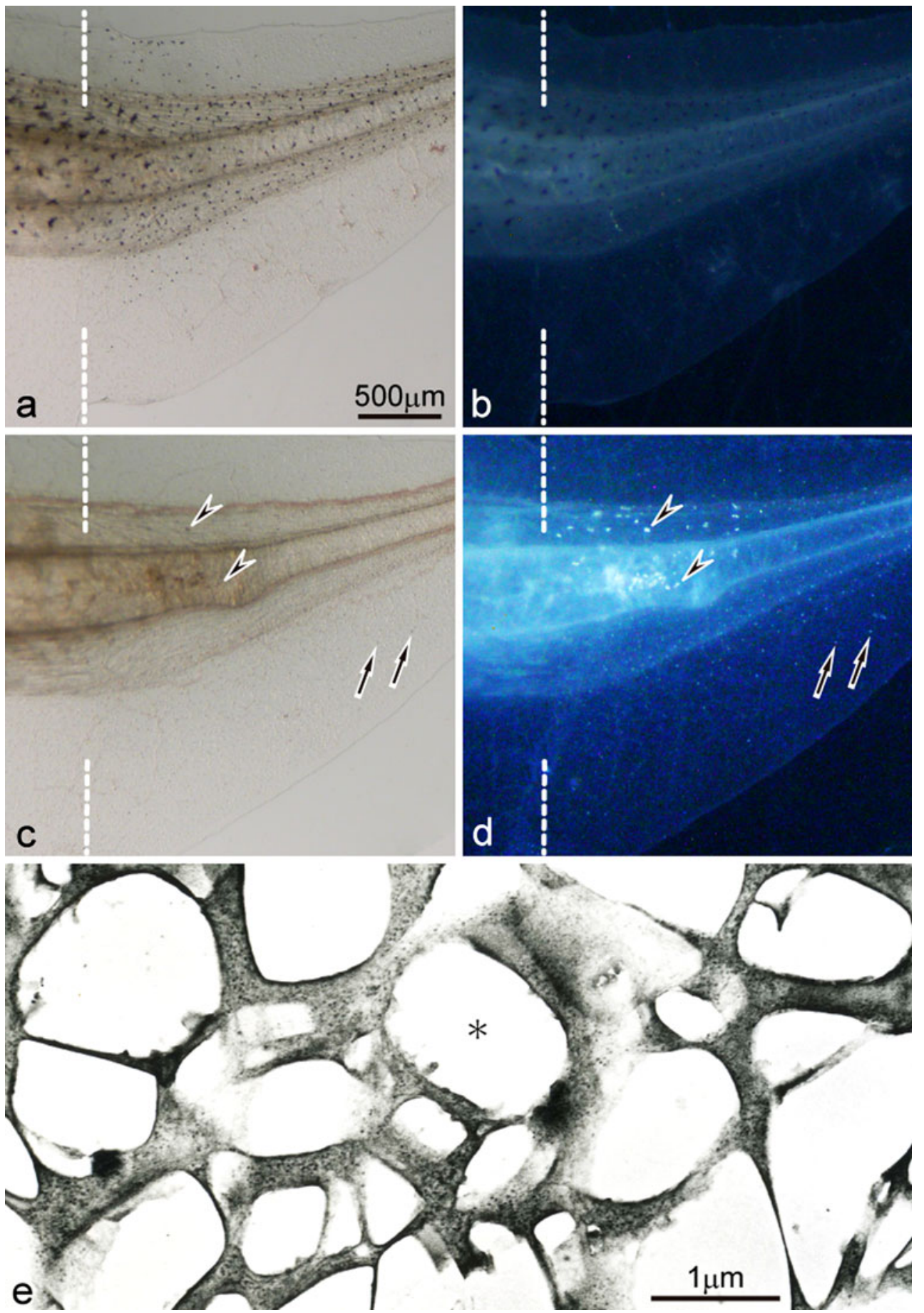

tail at least 2 days post-amputation, and their number gradually increased as the regenerating tail grew (Fig. 7). Melanophores appeared in the somites and fin in the wildtype regenerating tail, and their distribution was similar to that in the intact tadpole tail. Neither white pigment cells nor iridophores appeared in the wild-type regenerating tail.

In contrast, abundant white pigment cells, which were normally present in the posterior part of the mutant tadpole tail, appeared in the mutant regenerating tail (Fig. 6c, d). After amputation of the posterior half of the mutant tadpole tail, a small number of white pigment cells began to appear in the regenerating tail 2 or 3 days post-amputation, and their number gradually increased as the regenerating tail grew (Fig. 7). On days 5 and 6 post-amputation, the number of white pigment cells in the mutant regenerating tail was not statistically different from that of melanophores in the wild-type regenerating tail $(P>0.05)$ (Fig. 7). The localization and density of white pigment cells in the mutant regenerating tail were similar to those of melanophores in the wild-type regenerating tail (Fig. 6a-d). Dopa reaction was carried out to detect 


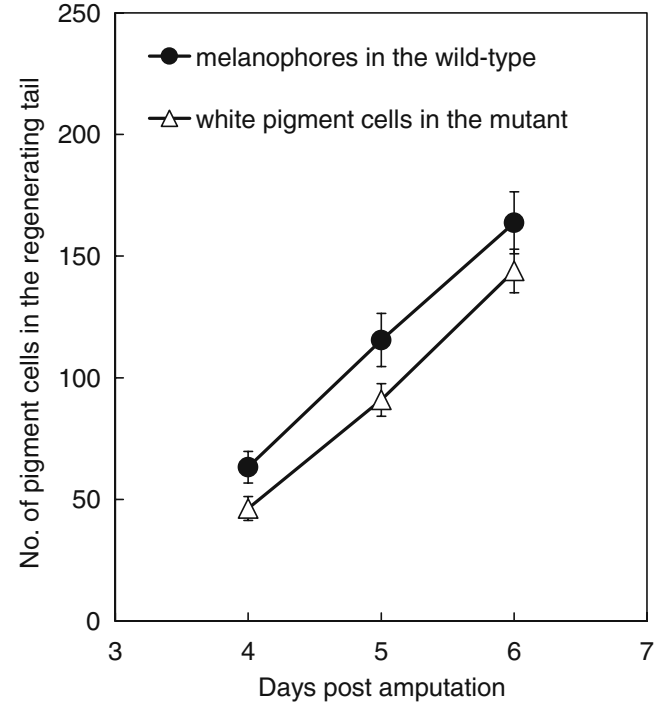

Fig. 7 The number of newly differentiated pigment cells in the regenerating tail of the wild-type and the mutant. After amputation of the posterior half of the tadpole tail (stage 48/49), melanophores or white pigment cells were counted in the regenerating tail of either the wild-type $(n=15)$ or the mutant $(n=16)$, respectively, on days 4,5 , and 6 post-amputation. The number of white pigment cells in the mutant regenerating tail was not statistically different from that of melanophores in the wild-type regenerating tail on days 5 and 6 post amputation ( $t$ test, $P>0.05)$

dopa-positive cells in the mutant regenerating tail (Fig. 8). The result showed that dopa staining was observed in white pigment cells (Fig. 8, arrowheads) as well as melanophore precursors (Fig. 8, arrows) in the mutant regenerating tail.

In addition to white pigment cells, a small number of iridophores, which were never present in the intact tadpole tail, began to appear from 5 to 6 days post-amputation in the mutant regenerating tail. In the 10-day mutant regenerating tail, about 10-30 iridophores appeared in the somites, although the exact number could not be counted because iridophores often overlapped each other. In the mutant regenerating tail, iridophores specifically appeared in the somites near the amputation level, while white pigment cells were present in both the somites and fin (Fig. 6c, d). Electron-microscopic observation demonstrated that iridophores in the mutant regenerating tail contained many reflecting platelets, which were irregular in size and shape (Fig. 6e).

In the 19-day mutant regenerating tail, differentiated iridophores were distinct from white pigment cells under a fluorescence stereomicroscope (Fig. 9). White pigment cells which were small and aggregated pigment organelles (Fig. 9a, b), looked white under incident light and emitted green fluorescence under blue light (Fig. 9c-e). On the other hand, iridophores which were large and dispersed pigment organelles (Fig. 9a, b), reflected light under incident light, but did not emit green fluorescence under blue light (Fig. 9c-e). White pigment cells in the mutant regenerating tail dispersed pigment organelles in response to $\alpha-\mathrm{MSH}$, whereas iridophores did not respond to $\alpha-\mathrm{MSH}$, or aggregated slightly, if any, in the presence of $\alpha-\mathrm{MSH}$ (data not shown).

\section{Discussion}

In the periodic albino mutant $\left(a^{p} / a^{p}\right)$ of Xenopus laevis, unusual pigment cells specifically appear which resemble leucophores in the fish, Oryzias latipes, with respect to color, blue light-induced fluorescence, and response to $\alpha$-MSH (Fukuzawa 2004). These cells were called "leucophore-like cells", although pigment organelles contained in these cells are ultrastructurally different from leucosomes in leucophores (Fukuzawa 2004). The author has reported that "leucophore-like cells" are unusual in (1) appearing white under incident light, (2) emitting green fluorescence under blue light, (3) exhibiting pigment dispersion in the presence
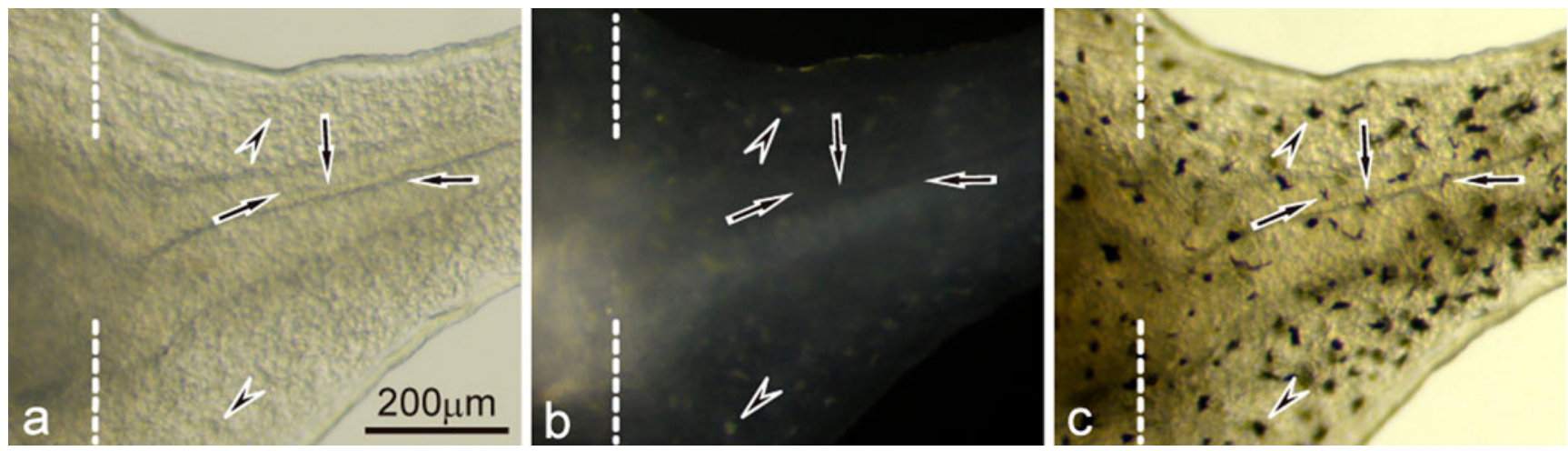

Fig. 8 Dopa staining in the 5-day regenerating tail in the mutant (amputated at stage 49). a,b The mutant regenerating tail before dopa staining observed under transmitted light (a), or incident light (b). c The mutant regenerating tail after dopa staining observed under transmitted light. Dashed lines indicate the amputation level. White pigment cells were appearing in the mutant regenerating tail on day 5 post-amputation (b). Dopa staining was observed in white pigment cells (arrowheads) as well as melanophore precursors which were not visible under transmitted light or incident light before dopa reaction (arrows) 
Fig. 9 Differentiation of iridophores and white pigment cells in the 19-day mutant regenerating tail (amputated at stage 50). a,b The mutant regenerating tail observed under transmitted light (a) or incident light (b). $\mathbf{c}-\mathbf{e}$ Enlarged view of the mutant regenerating tail of another tadpole observed under light (d), or blue light (e). Dashed lines indicate the amputation level. White pigment cells (arrows) which were small and aggregated pigment organelles, looked white under incident light and emitted green fluorescence under blue light. In contrast, iridophores (arrowheads) which were large and dispersed light under incident light, but did not emit green fluorescence under blue light transmitted light (c), incident pigment organelles, reflected

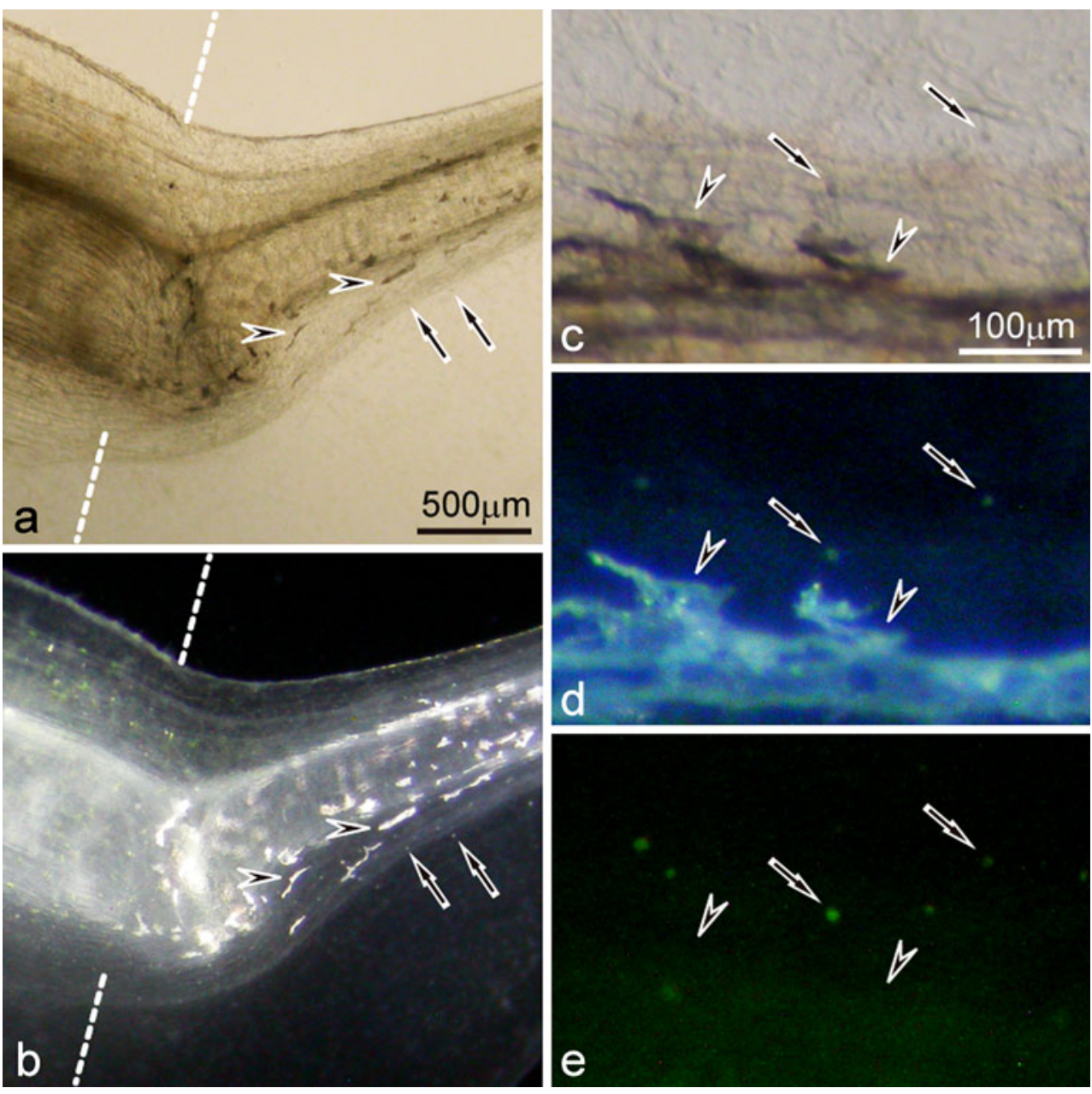

of $\alpha$-MSH, and (4) containing many reflecting platelet-like organelles (Fukuzawa 2004). However, the function of $a^{p}$ gene is not known. In this paper, "leucophore-like cells" in the periodic albino mutant are called "white pigment cells", because the term "leucophore" may be confusing or misleading in discussing the origin of these cells.

The Xenopus tadpole tail is best suited to investigate the relation between white pigment cells and melanophores, because only white pigment cells are present in the posterior part of the mutant tadpole tail, whereas only melanophores localize in the same region of the wild-type tadpole tail. Furthermore, the wild-type Xenopus tadpole tail has been recently utilized for regeneration research (Beck et al. 2009; Slack et al. 2008; Tseng and Levin 2008), and differentiation of neural crest derivatives has been investigated in the regenerating tail (Lin et al. 2007). In the present experiments, white pigment cells of the periodic albino mutant were compared with melanophores of the wild-type, using a cell culture system and a tailregenerating system.

White pigment cells in the periodic albino mutant are thought to derive from the melanophore lineage, because (1) white pigment cells are essentially similar to melano- phores of the wild-type with respect to their localization and density in the tadpole tail (Fig. 1), (2) white pigment cells respond to $\alpha-\mathrm{MSH}$ in the same way that melanophores do (Fig. 1), (3) white pigment cells contain premelanosomes that are observed in melanophore precursors (Fig. 5), and (4) white pigment cells show the characteristic features of both melanophores and iridophores (Fukuzawa 2004). As I discuss below, the present experiments provides further data to think about the mechanism as to how white pigment cells originate in the periodic albino mutant.

In the periodic albino mutant, melanophore precursors were maintained without differentiating into melanophores in the present culture condition using serum-free medium (Fig. 4), whereas wild-type melanophores differentiated in the same culture condition (Fig. 2). Indeed, mutant melanophore precursors at the late stage of development were discriminated from those at early to middle stages of development by dopa reaction (Fig. 3), combined dopapremelanin reaction (Fig. 3), and electron microscopy (Fig. 4). While the former cells contained stage II premelanosomes and a small number of partially melanized stage III melanosomes, the latter cells had stage I and II premelanosomes (Fig. 4). In our previous study, vesicles 
and early premelanosomes were also observed electron microscopically in wild-type Xenopus melanoblasts cultured in Steinberg's BSS (Fukuzawa and Ide 1988). Although melanoblast markers such as mitf (Lister et al. 1999; Goding 2000; Kumasaka et al. 2005), kit (Rawls and Johnson 2003; Slominski et al. 2004), and dct (Kelsh et al. 2000) have been utilized for the study of melanophore development, detection of pigment organelles is also useful to discuss pigment cell development (Bagnara et al. 1979a, b; Bagnara 1998). In the present study, ultrastructural observation revealed that white pigment cells in the periodic albino mutant contained organelles showing characteristics of melanophore precursors at various stages of development (Fig. 5). Some white pigment cells contained stage II premelanosomes which were characteristic of melanophore precursors at early to middle stages of development, while the other white pigment cells had stage II premelanosomes and a small number of partially melanized stage III melanosomes, which were characteristic of melanophore precursors at the late stage of development. Furthermore, the number of reflecting platelets contained in white pigment cells varied. Based upon the fact that white pigment cells are essentially similar to melanophores with respect to their localization, number, and response to $\alpha$-MSH, the present results support the possibility that white pigment cells arise from melanophore precursors at various stages of development and accumulate reflecting platelets characteristic of iridophores. Previous observation of white pigment cells which contained $a^{p}$-type melanosomes in the mutant tadpole (Fukuzawa 2004) can be interpreted in such a way that some white pigment cells arise from differentiated melanophores. The other possibility that white pigment cells arise from iridophores and accumulate premelanosomes is not likely, because (1) the cellular response of white pigment cells to $\alpha-\mathrm{MSH}$ is basically different from that of iridophores, and (2) iridophores never appear in the intact tadpole tail in either the wild-type or the mutant.

When the tadpole tail of wild-type X. laevis is amputated, a functionally competent new tail is regenerated, and abundant melanophores appear in the regenerating tail (Bagnara 1960; Lin et al. 2007). It has been reported that such melanophores in wild-type regenerating tails arise from pre-existing melanophore precursors (Lin et al. 2007). After amputation of the tadpole tail in the periodic albino mutant, abundant white pigment cells, instead of melanophores, appeared in the regenerating tail (Figs. 6 and 7). White pigment cells in the mutant regenerating tail are essentially similar to melanophores in the wild-type regenerating tail with respect to their localization (Fig. 6), number (Fig. 7), and response to $\alpha$-MSH. Furthermore, dopa staining was observed in both white pigment cells and melanophore precursors in the mutant regenerating tail (Fig. 8). Taken together, these data favor the possibility that white pigment cells in the mutant regenerating tail arise from pre-existing melanophore precursors and accumulate reflecting platelets.

In addition to white pigment cells, a small number of iridophores which were never present in the intact tadpole tail appeared in the somites near the amputation level in the mutant regenerating tail (Figs. 6 and 9). To my knowledge, this is the first demonstration that different types of pigment cells appeared following tail amputation. Iridophore appearance in the iridophore-free area of wild-type $X$. laevis has been demonstrated in the experiment where hypophyseal placode was removed at an early tail bud stage (Bagnara 1957). In the hypophysectomized embryos, iridophores appear in the tail fin, where melanophores normally localize and iridophores are totally absent (Bagnara 1957). The result of the hypophysectomized embryos can be explained by the effect of $\alpha-\mathrm{MSH}$, because iridophores are stimulated in the environment where $\alpha$-MSH is absent or scarce (Bagnara 1987). It is possible that insufficient $\alpha$-MSH may cause iridophore differentiation in the regenerating tail of the periodic albino mutant. However, the simple effect of $\alpha$-MSH cannot completely explain the following results: (1) iridophores appear only in the somites, but not in the fin, of the mutant regenerating tail (Figs. 6 and 9), whereas iridophores appear in the tail fin of the wild-type hypophysectomized larvae (Bagnara 1957), and (2) white pigment cells appear in the mutant regenerating tail (Figs. 6 and 9), but not in the wildtype hypophysectomized larvae (Bagnara 1957). Factor(s) other than $\alpha$-MSH may cause the expression of white pigment cells and iridophores in the mutant regenerating tail. Leukocyte tyrosine kinase $(L t k)$ has been recently suggested to function in iridophore specification in zebrafish (Lopes et al. 2008). It should be studied whether pigment cell expression is affected by $L t k$ in the periodic albino mutant of $X$. laevis. The function of mitf (reviewed by Goding 2000) should also be taken into consideration, since this gene is essential for the normal development of retinal pigment epithelium and melanophores (melanocytes), both of which are affected in the periodic albino mutant (Hoperskaya 1975). At this time, the mechanism is not clear as to how iridophores arise in the mutant regenerating tail. Iridophores may arise from pre-existing iridophore precursors (iridoblasts) or pluripotent stem cells in the mutant regenerating tail.

In the periodic albino mutant, melanophores begin to appear at stage 41 in the head region, and trunk melanophores are observed until stage 48 ; however, they gradually disappear in vivo. On the other hand, white pigment cells are detected at least from stage 45 in the mutant (Fukuzawa 2004). Recently, it has been demonstrated that there are two distinct melanophore lineages in the zebrafish embryo, namely, the embryonic or ontogenetic melanophore lineage, and the regenerative and metamorphic melanophore lineage (Hultman et al. 2009; Hultman and Johnson 2010). It is tempting to speculate on this point 
that early differentiating melanophores and late appearing white pigment cells derive from different melanophore lineages in the periodic albino mutant. The question also remains to be answered whether the melanophore lineage in the intact tadpole is different from that in the regenerating tail. Further studies are needed to clarify these points.

It has been reported that pigment organellogenesis is affected in all types of pigment cells in the periodic albino mutant (Fukuzawa 2006). Reflecting platelets of mutant iridophores were different from those of wild-type iridophores in size and shape (Figs. 2 and 4). Ultrastructural observation shows that reflecting platelets in white pigment cells are analogous to those in mutant iridophores (Figs. 4 and 5). White pigment cells which derive from the melanophore lineage in the mutant may have a propensity to form reflecting platelets characteristic of iridophores. It should be pointed out that white pigment cells are different from iridophores in size, shape, blue light inducedfluorescence, and response to $\alpha-\mathrm{MSH}$, although reflecting platelets are contained in both of them. The difference between white pigment cells and iridophores may reflect the different origin, that is, iridophores derive from iridoblasts, whereas white pigment cells arise from melanophore precursors and accumulate reflecting platelets characteristic of iridophores. It is possible that purines or other crystals are deposited in premelanosomes to form reflecting platelets in white pigment cells (Fig. 5f, arrows). Further analyses are necessary to understand how the phenotype of light-reflecting pigment cells is determined and how organellogenesis is controlled in pigment cells. Investigations of the periodic albino mutant of $X$. laevis would provide some insight for organellogenesis and development of various pigment cells.

Acknowledgments The author thanks Dr. J.T. Bagnara for his critical reading of the manuscript. This work was supported by Keio Gijuku Academic Development Funds.

Open Access This article is distributed under the terms of the Creative Commons Attribution Noncommercial License which permits any noncommercial use, distribution, and reproduction in any medium, provided the original author(s) and source are credited.

\section{References}

Bagnara JT (1957) Hypophysectomy and the tail darkening reaction in Xenopus. Proc Soc Exp Biol Med 94:572-575

Bagnara JT (1960) Tail melanophores of Xenopus in normal development and regeneration. Biol Bull 118:1-8

Bagnara JT (1987) The neural crest as a source of stem cells. In: Maderson P (ed) Developmental and evolutionary aspects of the neural crest. Wiley, New York, pp 57-87

Bagnara JT (1998) Comparative anatomy and physiology of pigment cells in nonmammalian tissues. In: Nordlund JJ, Boissy RE, Hearing VJ, King RA, Ortonne J-P (eds) The pigmentary system: physiology and pathophysiology. Oxford University Press, New York, pp 9-40

Bagnara JT, Hadley ME (1973) Chromatophores and color change: the comparative physiology of animal pigmentation. Prentice-Hall, Englewood Cliffs, NJ

Bagnara JT, Matsumoto J, Ferris W, Frost SK, Turner WA Jr, Tchen TT, Taylor JD (1979a) Common origin of pigment cells. Science 203:410-415

Bagnara JT, Turner WA Jr, Rothstein J, Ferris W, Taylor JD (1979b) Chromatophore organellogenesis. In: Klaus SN (ed) Pigment cell, vol 4. S. Karger, Basel, pp 13-27

Beck CW, Belmonte JCI, Christen B (2009) Beyond early development: Xenopus as an emerging model for the study of regenerative mechanisms. Dev Dyn 238:1226-1248

Elworthy S, Lister JA, Carney TJ, Raible DW, Kelsh RN (2003) Transcriptional regulation of mitfa accounts for the sox10 requirement in zebrafish melanophore development. Development 130:2809-2818

Fukamachi S, Wakamatsu Y, Mitani H (2006) Medaka double mutants for color interfere and leucophore free: characterization of the xanthophore-somatolactin relationship using the leucophore free gene. Dev Genes Evol 216:152-157

Fukuzawa T (2004) Unusual leucophore-like cells specifically appear in the lineage of melanophores in the periodic albino mutant of Xenopus laevis. Pigment Cell Res 17:252-261

Fukuzawa T (2006) Abnormal pigment organellogenesis in iridophores and xanthophores of the periodic albino mutant of Xenopus laevis as shown in the neural tube culture system. Hiyoshi Rev Nat Sci Keio Univ 40:15-32

Fukuzawa T, Ide H (1986) Further studies on the melanophores of periodic albino mutant of Xenopus laevis. J Embryol Exp Morphol 91:65-78

Fukuzawa T, Ide H (1987) Melanophore differentiation in the periodic albino mutant of Xenopus laevis. Pigment Cell Res 1:197-201

Fukuzawa T, Ide H (1988) A ventrally localized inhibitor of melanization in Xenopus laevis skin. Dev Biol 129:25-36

Fukuzawa T, Bagnara JT (1989) Control of melanoblast differentiation in amphibia by $\alpha$-melanocyte stimulating hormone, a serum melanization factor, and a melanization inhibiting factor. Pigment Cell Res 2:171-181

Goding CR (2000) Mitf from neural crest to melanoma: signal transduction and transcription in the melanocyte lineage. Genes Dev 14:1712-1728

Hall BK, Hörstadius S (1988) The neural crest. Oxford University Press, London

Hirobe T (1984) Histochemical survey of the distribution of the epidermal melanoblasts and melanocytes in the mouse during fetal and postnatal periods. Anat Rec 208:589-594

Hirobe T, Takeuchi T (1977) Induction of melanogenesis in the epidermal melanoblasts of newborn mouse skin by MSH. J Embryol Exp Morphol 37:79-90

Hoperskaya OA (1975) The development of animals homozygous for a mutation causing periodic albinism $\left(a^{p}\right)$ in Xenopus laevis. $\mathrm{J}$ Embryol Exp Morphol 34:253-264

Hoperskaya OA (1981) Induction - The main principle of melanogenesis in early development. Differentiation 20:104-116

Hultman KA, Johnson SL (2010) Differential contribution of directdeveloping and stem cell-derived melanocytes to the zebrafish larval pigment pattern. Dev Biol 337:425-431

Hultman KA, Budi EH, Teasley DC, Gottlieb AY, Parichy DM, Johnson SL (2009) Defects in ErbB-dependent establishment of adult melanocyte stem cells reveal independent origins for embryonic and regeneration melanocytes. PLoS Genet 5:e1000544

Jones KY, Elsdale TR (1963) The culture of small aggregates of amphibian embryonic cells in vitro. J Embryol Exp Morphol 11:135154 
Kelsh RN, Schmid B, Eisen JS (2000) Genetic analysis of melanophore development in zebrafish embryos. Dev Biol 225:277-293

Kelsh RN, Harris ML, Colanesi S, Erickson CA (2009) Stripes and belly-spots - A review of pigment cell morphogenesis in vertebrates. Semin Cell Dev Biol 20:90-104

Kumasaka M, Sato S, Yajima I, Goding CR, Yamamoto H (2005) Regulation of melanoblast and retinal pigment epithelium development by Xenopus laevis Mitf. Dev Dyn 234:523-534

Le Douarin NM, Kalcheim C (1999) The neural crest. Cambridge University Press, Cambridge

Lin G, Chen Y, Slack JMW (2007) Regeneration of neural crest derivatives in the Xenopus tadpole tail. BMC Dev Biol 7:56

Lister JA, Robertson CP, Lepage T, Johnson SL, Raible DW (1999) nacre encodes a zebrafish microphthalmia-related protein that regulates neural-crest-derived pigment cell fate. Development 126:3757-3767

Lister JA, Cooper C, Nguyen K, Modrell M, Grant K, Raible DW (2006) Zebrafish foxd3 is required for development of a subset of neural crest derivatives. Dev Biol 290:92-104

Lopes SS, Yang X, Muller J, Carney TJ, McAdow AR, Rauch GJ, Jacoby AS, Hurst LD, Delfino-Machin M, Haffter P, Geisler R, Johnson SL, Ward A, Kelsh RN (2008) Leukocyte tyrosine kinase functions in pigment cell development. PLoS Genet 4:e1000026

MacMillan GJ (1979) An analysis of pigment cell development in the periodic albino mutant of Xenopus. J Embryol Exp Morphol $52: 165-170$

MacMillan GJ (1981) Experimental evidence for autonomous action of the periodic albinism $\left(a^{p}\right)$ gene within developing retinal pigment cells and melanophores of Xenopus laevis. J Embryol Exp Morphol 64:333-341

MacMillan GJ, Gordon AM (1981) Iridophore development in wildtype and periodic albino Xenopus larvae. Experientia 37:183184

Minchin JEN, Hughes SM (2008) Sequential actions of Pax3 and $\operatorname{Pax} 7$ drive xanthophore development in zebrafish neural crest. Dev Biol 317:508-522
Mishima Y (1960) New technic for comprehensive demonstration of melanin, premelanin, and tyrosinase sites. J Invest Dermatol 34:355-360

Nieuwkoop PD, Faber J (1967) Normal table of Xenopus laevis (Daudin). North-Holland, Amsterdam

Parichy DM, Rawls JF, Pratt SJ, Whitfield TT, Johnson SL (1999) Zebrafish sparse corresponds to an orthologue of c-kit and is required for the morphogenesis of a subpopulation of melanocytes, but is not essential for hematopoiesis or primordial germ cell development. Development 126:3425-3436

Parichy DM, Mellgren EM, Rawls JF, Lopes SS, Kelsh RN, Johnson SL (2000a) Mutational analysis of endothelin receptor b1 (rose) during neural crest and pigment pattern development in the zebrafish Danio rerio. Dev Biol 227:294-306

Parichy DM, Ransom DG, Paw B, Zon LI, Johnson SL (2000b) An orthologue of the kit-related gene $f m s$ is required for development of neural crest-derived xanthophores and a subpopulation of adult melanocytes in the zebrafish, Danio rerio. Development 127:3031-3044

Raposo G, Marks MS (2007) Melanosomes - dark organelles enlighten endosomal membrane transport. Nat Rev Mol Cell Biol 8:786-797

Rawls JF, Johnson SL (2003) Temporal and molecular separation of the kit receptor tyrosine kinase's roles in zebrafish melanocyte migration and survival. Dev Biol 262:152-161

Seldenrijk R, Huijsman KGH, Heussen AMA, van de Veerdonk FCG (1982) A comparative ultrastructural and physiological study on melanophores of wild-type and periodic albino mutants of Xenopus laevis. Cell Tissue Res 222:1-9

Slack JMW, Lin G, Chen Y (2008) The Xenopus tadpole: a new model for regeneration research. Cell Mol Life Sci 65:54-63

Slominski A, Tobin DJ, Shibahara S, Wortsman J (2004) Melanin pigmentation in mammalian skin and its hormonal regulation. Physiol Rev 84:1155-1228

Tseng AS, Levin M (2008) Tail regeneration in Xenopus laevis as a model for understanding tissue repair. J Dent Res 87:806-816 